\title{
A Formal Approach To Validate Block-Chains
}

\author{
Roobaea Alroobaea ${ }^{1}$
}

\begin{abstract}
Our goal is to propose a suitable approach for validating blockchains. For this purpose, we intend to adopt formal methods which are based on strong mathematical foundations. More precisely, we follow a model-based testing approach. The latter consists in describing the behavior of the system using a specific formalism, deriving test cases from the obtained model and then executing the obtained tests on the implementation to check whether it is correct or not. The adopted formalism corresponds to the timed automaton Model. The generated tests may be either digital or analog. Moreover, we propose several techniques which allow to solve the state explosion which may be encountered during the verification and test generation phases.
\end{abstract}

Index Terms-BlockChains, Formal Methods, Model Based Testing, Generation, Execution.

\section{INTRODUCTION}

Blockchains correspond to a new technology which is becoming more and more famous nowadays. It is mainly used for the establishment of new crypto-currencies (like bitcoins) and for electronic money transactions. [1] define it as "a distributed ledger shared between nodes in a peerto-peer network. Basically, a ledger is simply a database that is maintained and updated by every node in the network". The Blockchain is not a new technology and it combines four types of technology to enable it and build a ledger. These technologies are peer to peer network, Merkle trees, hashes and public/private key cryptosystem. There are a lot of applications for Blockchain, such as , Cryptocurrencies, Healthcare, Supply Chain Management and Cybersecurity [2]. Also, it can be used in Internet of Things (IoT) such as Smart Homes and cites [3]. Furthermore, it can be integrated with another technology to store and control the transaction such as fog computing and cloud computing [4], [5].

Our goal in this work is to validate blockchains by adopting formal methods based on sophisticated mathematical techniques. For that purpose, we opt for a modelbased testing approach which consists in deriving test cases automatically from a formal description of the blockchain behavior. In Section II, a brief overview about blockchains will be given. In Section III, the notion of formal methods will be introduced. In Section IV, some challenges related to blockchains testing will be we listed. In Section V, the main steps for validating blockchains following a model-based approach will be presented. Finally, Section VI concludes the paper.$$
\begin{gathered}
\text { College of CIT, Taif University, Saudi Arabia. } \\
\text { r.robaidtu.edu.sa }
\end{gathered}
$$

\section{About Blockchains}

The Blockchain idea was first introduced in 1991. It is a chain of sequential information that is constantly updated. The information is grouped into blocks, which are generated gradually from the resulting data. From each block, a hash function calculates a hash value, which in turn is inserted at the beginning of the next block. To prove the integrity of the chain, all that is needed is to recalculate the hash values for each block and compare the final score to the published value. Manipulations of the chain are therefore immediately apparent during the test [6].

The way blockchain looks in practice differs depending on the implementation - and the combination of techniques and methodologies in conjunction with the blockchain. Therefore, cryptocurrencies and smart contracts and system architecture security are discussed below [7].

The Blockchain idea can be used to create and manage a decentralized and anonymous currency such as Bitcoin. In the Bitcoin system, computers around the world maintain identical copies of the same block chain, which records all financial transactions based on that currency. Once a Bitcoin transfer is made, it is distributed via peer-to-peer to store it in the world famous blockchain [8].

From the published bank transfer credentials, anyone can create and republish a new block, so the blockchain is continually being extended. If you want to update the blockchain you have to solve a cryptographic puzzle: The puzzle states that the hash value must start at the beginning of a block with a defined number of zeros. Such a hash value can only be found by adding a matching code to the transfer information in the new block of the chain. The search for a corresponding code is called scouring or mines. This is intentionally very elaborate, requires the use of enormous amounts of energy and is therefore as the model of mining very resource hungry. In 2014 alone, mining required so much electricity worldwide that Ireland could have been completely supplied with it. As an automatic reward built into the system, the successful prospector of a block receives new bitcoins. Who creates a block, thus also coins new coins [9].

Bitcoin is not the only cryptocurrency based on blockchain. A handful of Altcoins (alternative coins) have managed to pick up on the Bitcoin idea and expand it significantly. Of particular note is Ethereum, a currency more likely to be more interesting to developers than Bitcoin, which offers the ability to create smart contracts and do more than just write simple scripts [10].

Ethereum significantly reduces the write delay of the 
information compared to Bitcoin. If the miners in the Bitcoin system create a new block only about every ten minutes, the Ethereum miners add a new block to the chain every twelve seconds. In addition, Ethereum adds an account concept. Accounts can be charged with the Bitcoin equivalent of ether. A specific account - called the zero account - is called to create a smart contract. This Smart Contract then has the amount of Ether that should be transferred with the transaction and can be automatically triggered by external events. If, for example, a specific situation occurs and the smart contract is deemed to have been fulfilled, the credit is typically transferred to a predefined account. However, there are also no-money smart contracts that send messages to other smart contracts and trigger a response [11].

The crypto-currencies need protection against such attacks, where a participant claims to be a large number of different participants, jeopardizing the consensus-based integrity of the decentralized network. At Bitcoin, miners have to use power to participate in the infrastructure - and an individual can never have that much power and computing resources to mimic more than half of the world's miners. This approach is called Proof of Work. In the case of Ethereum, the inventors are planning a new model that appeals to the self-interest of the participants. Accordingly, the right to coinage would be paid by paying existing Ethereum coins (ethers). This model is based on the assumption that no investor wants to endanger the integrity of a system in which he has invested himself - this would jeopardize the value of the entire currency and thus its capital [12], [11].

\section{Challenges for Testing}

This development has major consequences for testers. The test professional will have to do his work differently than now. There are a number of reasons for this:

- A programming error in a Self Executing Contract leads faster than now to a test blocking finding. We can no longer intervene manually within the process.

- Stricter than now, attention must be paid in the test preparation to the creation, reading, updating and deletion (CRUD) of test data during test execution. Test data cannot be changed as quickly anymore. A test network is usually used for testing.

- Transaction can initially be approved, but can be rejected retroactively in a subsequent block. Bitcoin uses the rule to wait at least two blocks before you can assume that the transaction has been approved.

- Authorization who is allowed to view and change which data is now done by the blockchain whereby it is checked whether the correct encryption key is supplied. This depends on the implementation, the foreign or the private key. The authorization layer in the front application must take this into account. Certainly if data is supplied through the OTAP street when software is delivered.

- It becomes even more complex when data from other block chains is consulted. At the moment, however, it is unclear whether the dynamics of periodically making a block definitive allow this kind of direction at all.

On the other hand, the work of testers also becomes easier on a number of points. A number of test types are no longer necessary for the blockchain itself, unless it is a private blockchain. In the latter case, checking whether the architecture has been put in place is important. If we assume that a public blockchain is being used, then it seems to be agreed that:

- Performance testing is given a completely different meaning. The performance is partly determined by the speed of the network. In part, the performance is determined by the method of approval of the transactions.

- Failover test from one database to another is not necessary, because the blockchain is distributed. When a node in the network fails, there is simply less capacity available, the database as such remains in operation.

- Fallback test is not necessary if the different nodes are geographically dispersed.

- Security testing on the database becomes more limited because all data is stored encrypted and can only be read with a foreign or private key, depending on the implementation. The strength of the encryption is enforced in the code of the blockchain.

Functional testing of the Self Executing Contract is of course always necessary, whether it is a public or private blockchain, just like the applications with which the data in the blockchain can be consulted and changed. Some changes will always remain manual, such as contract termination because the customer goes to another supplier. There are also applications that will continue to be intentionally done with human intervention to prevent someone from selling a valuable object such as his house under pressure [13], [14].

The challenges of functional testing of a system using blockchains are fully valid for other test types such as the User Acceptance Test, the Usability Test, the Security Test, and the Production Acceptance Test of the chain as a whole.

\section{Formal Methods}

Formal methods can be used to calculate whether a system description (specification) is inherently consistent, whether certain properties can be guaranteed, and whether the requirements have been properly designed and implemented (currently only theoretical). Furthermore, the specification can be used for simulation / animation, for code generation, for test generation and test evaluation [15]. The extra effort to describe something formally must have a justification. Just formalizing to get a formal specification is no justification. A formal specification is not meaningful at all costs and for all parts of a system. Non-safety-critical parts do not necessarily have to be formally described. For each formal component, it must be clear in which analyzes and constructions the formalization is used, or if it is intended as a documentation, it should be clear who should read it (can he read it?). If this is not clearly defined, the effort for a formal specification is not worthwhile. 
A development with formal methods differs from a traditional development in several points. To explain these points, one must first distinguish two varieties of formal specification: operational and axiomatic.

In an operational specification, only what is explicitly specified happens (as in an imperative programming language). In an axiomatic specification, anything can happen that has not been explicitly excluded. An operational specification describes the function of the system, while an axiomatic specification describes the properties.

\section{A. Timed Automata}

Timed Automata (TA) [16], [17] correspond to a simple graphical formalism for describing the behaviour of wide classes of systems which combine continuous and discrete aspects.

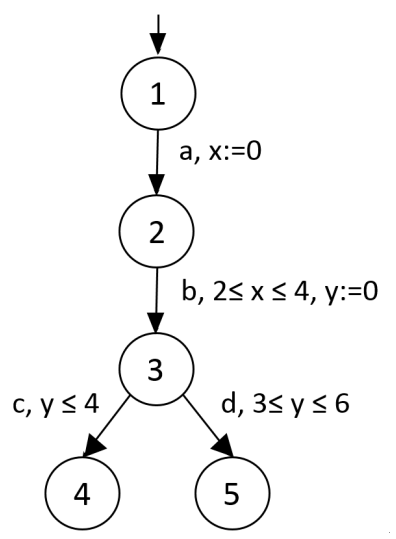

Fig. 1. A timed automaton with 5 states, 4 transitions, 4 actions and 2 clocks.

In Figure 1 we give an example of a timed automaton which has 5 nodes, 4 transitions, 4 actions and 2 clocks.

\section{B. Different Types of Testers}

One may distinguish between different types of tests:

- TA Testers: presented as a TA.

- On-the-Fly Analog Testers: measure time with precision and are generated during the test execution phase.

- Digital Testers: represented as trees and use numerical clocks to estimate time.

\section{Conformance Relations}

For untimed systems we consider the conformance relation ioco [18] and for timed systems we could adopt the relation tioco [19], [20], [21] which allows to compare timed specifications and implementations.

\section{PRoposed Approach}

Our goal is to follow a Model-Based Testing methodology to validate block-chains. Next we give a brief overview about the main steps of this approach.

\section{A. Model Specification}

The First challenge corresponds to the fact of providing a suitable Model for the specification of the blockchain to test. The proposed model should be written within an adequate formalism. For instance, we may adopt the model of Timed Automata as in [21], [22].

\section{B. Test Generation}

We get inspired by the generation algorithm of [23]. The later constructs a test case in the form of a tree. The proposed test generation methodology is based on model checking. The basic principle is to encode test generation as a reachability analysis that can be tackled using the tool UPPAAL [24].

\section{Test Selection and Execution}

Selection techniques help to reduce the number of generated test scenarios. For that purpose, we need to consider specific coverage criteria like states coverage, actions coverage, locations coverage etc. Concerning test execution we need to adopt a standard-based platforms like TTCN3 [25], [26], [27]. In addition, we may need to consider appropriate test isolation policies which allow to avoid interference between business functionalities and test cases as proposed in the work of [28].

\section{Test Component Placement}

The task here consists in optimizing the placement of the different test components. For this goal, we are inspired by the work of [29] from which we identify the following ingredients.

- Different Constraints:

\begin{tabular}{|l|l|}
\hline Constraint Type & References \\
\hline \hline Network Constraints & {$[30][31]$} \\
\hline Energy Constraints & {$[32][33]$} \\
\hline Application Constraints & {$[34]$} \\
\hline Node Constraints & {$[35]$} \\
\hline
\end{tabular}

- Objective Functions:

\begin{tabular}{|l|l|}
\hline Objective Function & References \\
\hline \hline QoS-assurance & {$[30][36]$} \\
\hline Cost & {$[35][37]$} \\
\hline Energy & {$[32][38]$} \\
\hline Migrations & {$[39][40]$} \\
\hline $\begin{array}{l}\text { Network Delay and } \\
\text { Execution Time }\end{array}$ & {$[31]$} \\
\hline
\end{tabular}


- Algorithms:

\begin{tabular}{|l|l|}
\hline Algorithm & References \\
\hline \hline $\begin{array}{l}\text { Search-based } \\
\text { Algorithms }\end{array}$ & {$[41][34]$} \\
\hline Dynamic Programming & {$[33][42]$} \\
\hline Genetic Algorithms & {$[43][44]$} \\
\hline Game Theory & {$[45]$} \\
\hline Deep Learning & {$[46]$} \\
\hline Complex networks & {$[40]$} \\
\hline $\begin{array}{l}\text { Mathematical } \\
\text { Programming }\end{array}$ & {$[31]$} \\
\hline
\end{tabular}

\section{E. Solving State Explosion Problem}

Formal methods usually suffer from state explosion problem which means obtaining a state graph which is huge and difficult to manipulate. Next, we list some solutions to deal with this problem.

- For formal verification:

\begin{tabular}{|l|l|}
\hline Technique & References \\
\hline \hline Abstraction & {$[47][48]$} \\
\hline $\begin{array}{l}\text { Modularization and } \\
\text { Compositionality }\end{array}$ & {$[49][50]$} \\
\hline Symmetry Detection & {$[51][52][53]$} \\
\hline $\begin{array}{l}\text { Data Independence De- } \\
\text { tection }\end{array}$ & {$[54][55]$} \\
\hline $\begin{array}{l}\text { Removing Functional } \\
\text { Dependencies }\end{array}$ & {$[56][57]$} \\
\hline Using Reversible Rules & {$[58][59]$} \\
\hline
\end{tabular}

- For Model-Based Testing:

\begin{tabular}{|l|l|}
\hline Technique & References \\
\hline \hline Refinement Methods & {$[60]$} \\
\hline $\begin{array}{l}\text { Diminishing the Size of } \\
\text { Digital Tests }\end{array}$ & {$[61]$} \\
\hline Generating TA Testers & {$[62][63][61]$} \\
\hline $\begin{array}{l}\text { Updating Test Cases af- } \\
\text { ter System Evolution }\end{array}$ & {$[64]$} \\
\hline $\begin{array}{l}\text { Resource Aware Test } \\
\text { Component Placement }\end{array}$ & {$[65][66]$} \\
\hline State Identification & {$[67][68][69]$} \\
\hline
\end{tabular}

\section{CONCLUSION}

Our work is at its beginning and many tasks have to be achieved in the near future. We need to choose adequate formalism with adequate tools. Then we need to identify the specific aspects to model in order to keep the size of the obtained formal specification and to avoid the space explosion problem from which formal methods suffer in general. Moreover, we will need to implement the proposed approach and to validate it experimentally. We may also work on combining functional and load aspects as proposed in [70], [71], [72], [73], [74], [75].

\section{REFERENCES}

[1] O. Cheikhrouhou and A. Koubaa, "Blockloc: Secure localization in the internet-of-things using blockchain," arXiv preprint arXiv:1904.13138, 2019.

[2] C. Jaikaran, Blockchain: Background and policy issues. Congressional Research Service, 2018.

[3] T. Alam, "Blockchain and its role in the internet of things (iot)," arXiv preprint arXiv:1902.09779, 2019.

[4] _ - "Iot-fog: A communication framework using blockchain in the internet of things," arXiv preprint arXiv:1904.00226, 2019.

[5] T. Alam and M. Benaida, "The role of cloud-manet framework in the internet of things (iot)," arXiv preprint arXiv:1902.09436, 2019.

[6] M. Pilkington, "11 blockchain technology: principles and applications," Research handbook on digital transformations, vol. 225, 2016.

[7] R. Zhang, R. Xue, and L. Liu, "Security and privacy on blockchain," arXiv preprint arXiv:1903.07602, 2019.

[8] L. Hughes, Y. K. Dwivedi, S. K. Misra, N. P. Rana, V. Raghavan, and V. Akella, "Blockchain research, practice and policy: Applications, benefits, limitations, emerging research themes and research agenda," International Journal of Information Management, vol. 49, pp. 114129, 2019.

[9] D. Yaga, P. Mell, N. Roby, and K. Scarfone, "Blockchain technology overview," arXiv preprint arXiv:1906.11078, 2019.

[10] A. Kosba, A. Miller, E. Shi, Z. Wen, and C. Papamanthou, "Hawk: The blockchain model of cryptography and privacy-preserving smart contracts," in 2016 IEEE symposium on security and privacy (SP). IEEE, 2016, pp. 839-858.

[11] Y. Sompolinsky and A. Zohar, "Secure high-rate transaction processing in bitcoin," in International Conference on Financial Cryptography and Data Security. Springer, 2015, pp. 507-527.

[12] A. Higbee, "The role of crypto-currency in cybercrime," Computer Fraud \& Security, vol. 2018, no. 7, pp. 13-15, 2018.

[13] C.-F. Liao, C.-J. Cheng, K. Chen, C.-H. Lai, T. Chiu, and C. WuLee, "Toward a service platform for developing smart contracts on blockchain in bdd and tdd styles," in 2017 IEEE 10th Conference on Service-Oriented Computing and Applications (SOCA). IEEE, 2017, pp. 133-140.

[14] S. Stupar, M. B. Ćar, and E. Šahić, "Challenges of applying blockchain technology," in International Conference New Technologies, Development and Applications. Springer, 2019, pp. 355-364.

[15] M. Krichen, M. Lahami, O. Cheikhrouhou, R. Alroobaea, and A. J. Maâlej, "Security testing of internet of things for smart city applications: A formal approach," in Smart Infrastructure and Applications. Springer, 2020, pp. 629-653.

[16] R. Alur and D. Dill, "A theory of timed automata," Theoretical Computer Science, vol. 126, pp. 183-235, 1994.

[17] J. Sifakis and S. Yovine, "Compositional specification of timed systems," in 13th Annual Symposium on Theoretical Aspects of Computer Science, STACS'96, ser. LNCS, vol. 1046. Spinger-Verlag, 1996.

[18] J. Tretmans, "A Formal Approcah to Conformance Testing," Ph.D. dissertation, University of Twente, Twente, The Netherlands, 1992.

[19] M. Krichen, "Model-based testing for real-time systems," Ph.D. dissertation, PhD thesis, Universit Joseph Fourier (December 2007), 2007.

[20] M. Krichen and S. Tripakis, "Interesting properties of the conformance relation tioco," in ICTAC'06, 2006.

[21] M. Krichen, "A formal framework for black-box conformance testing of distributed real-time systems," International Journal of Critical Computer-Based Systems, vol. 3, no. 1/2, pp. 26-43, 2012.

[22] M. Krichen, O. Cheikhrouhou, M. Lahami, R. Alroobaea, and A. Jmal Maâlej, "Towards a model-based testing framework for the security of internet of things for smart city applications," in Smart Societies, Infrastructure, Technologies and Applications, R. Mehmood, B. Bhaduri, I. Katib, and I. Chlamtac, Eds. Cham: Springer International Publishing, 2018, pp. 360-365.

[23] J. Tretmans, "Testing concurrent systems: A formal approach," in CONCUR'99 Concurrency Theory, J. C. M. Baeten and S. Mauw, Eds. Berlin, Heidelberg: Springer Berlin Heidelberg, 1999, pp. 4665.

[24] G. B. qnd Alexandre David and K. G. Larsen, "A tutorial on uppaal," in International School on Formal Methods for the Design of Computer, Communication, and Software Systems, SFM-RT 2004. Revised Lectures, ser. LNCS, M. Bernardo and F. Corradini, Eds., vol. 3185. Springer Verlag, 2004, pp. 200-237. [Online]. Available: http://doc.utwente.n1/51010/ 
[25] M. Lahami, F. Fakhfakh, M. Krichen, and M. Jmaïel, "Towards a TTCN-3 Test System for Runtime Testing of Adaptable and Distributed Systems," in Proceedings of the 24th IFIP WG 6.1 International Conference Testing Software and Systems (ICTSS'12), 2012, pp. 71-86.

[26] M. Lahami, M. Krichen, and M. Jmaïel, "Safe and Efficient Runtime Testing Framework Applied in Dynamic and Distributed Systems," Science of Computer Programming (SCP), vol. 122, no. C, pp. 1-28, 2016.

[27] M. Krichen, R. Alroobaea, and M. Lahami, "Towards a runtime standard-based testing framework for dynamic distributed information systems," 2019.

[28] M. Lahami and M. Krichen, "Test Isolation Policy for Safe Runtime Validation of Evolvable Software Systems," in Proceedings of the 22nd IEEE International Conference on Enabling Technologies: Infrastructure for Collaborative Enterprises (WETICE'13), 2013, pp. 377-382.

[29] A. Brogi, S. Forti, C. Guerrero, and I. Lera, "How to place your apps in the fog - state of the art and open challenges," CoRR, vol. abs/1901.05717, 2019.

[30] R. Mahmud, K. Ramamohanarao, and R. Buyya, "Latency-aware application module management for fog computing environments," ACM Trans. Internet Technol., vol. 19, no. 1, pp. 9:1-9:21, Nov. 2018. [Online]. Available: http://doi.acm.org/10.1145/3186592

[31] V. B. C. Souza, W. Ramrez, X. Masip-Bruin, E. Marn-Tordera, G. Ren, and G. Tashakor, "Handling service allocation in combined fog-cloud scenarios," in 2016 IEEE International Conference on Communications (ICC), May 2016, pp. 1-5.

[32] M. Barcelo, A. Correa, J. Llorca, A. M. Tulino, J. L. Vicario, and A. Morell, "Iot-cloud service optimization in next generation smart environments," IEEE Journal on Selected Areas in Communications, vol. 34, no. 12, pp. 4077-4090, Dec 2016.

[33] V. B. Souza, X. Masip-Bruin, E. Marin-Tordera, W. Ramirez, and S. Sanchez, "Towards distributed service allocation in fog-to-cloud (f2c) scenarios," in 2016 IEEE Global Communications Conference (GLOBECOM), Dec 2016, pp. 1-6.

[34] C. Guerrero, I. Lera, and C. Juiz, "A lightweight decentralized service placement policy for performance optimization in fog computing," Journal of Ambient Intelligence and Humanized Computing, vol. 10, no. 6, pp. 2435-2452, Jun 2019. [Online]. Available: https://doi.org/10.1007/s12652-018-0914-0

[35] H. R. Arkian, A. Diyanat, and A. Pourkhalili, "Mist: Fog-based data analytics scheme with cost-efficient resource provisioning for iot crowdsensing applications," Journal of Network and Computer Applications, vol. 82, pp. 152 - 165, 2017. [Online]. Available: http://www.sciencedirect.com/science/article/pii/S1084804517300188

[36] S. Venticinque and A. Amato, "A methodology for deployment of iot application in fog," Journal of Ambient Intelligence and Humanized Computing, vol. 10, no. 5, pp. 1955-1976, May 2019. [Online]. Available: https://doi.org/10.1007/s12652-018-0785-4

[37] S. Wang, M. Zafer, and K. K. Leung, "Online placement of multicomponent applications in edge computing environments," IEEE Access, vol. 5, pp. 2514-2533, 2017.

[38] Z. Huang, K.-J. Lin, S.-Y. Yu, and J. Y. jen Hsu, "Colocating services in iot systems to minimize the communication energy cost," Journal of Innovation in Digital Ecosystems, vol. 1, no. 1, pp. 47 - 57, 2014. [Online]. Available: http://www.sciencedirect.com/science/article/pii/S2352664515000061

[39] B. Ottenwälder, B. Koldehofe, K. Rothermel, and U. Ramachandran, "Migcep: Operator migration for mobility driven distributed complex event processing," in Proceedings of the 7th ACM International Conference on Distributed Event-based Systems, ser. DEBS '13. New York, NY, USA: ACM, 2013, pp. 183-194. [Online]. Available: http://doi.acm.org/10.1145/2488222.2488265

[40] S. Filiposka, A. Mishev, and K. Gilly, "Community-based allocation and migration strategies for fog computing," in 2018 IEEE Wireless Communications and Networking Conference (WCNC), April 2018, pp. 1-6.

[41] H. Gupta, A. V. Dastjerdi, S. K. Ghosh, and R. Buyya, "ifogsim: A toolkit for modeling and simulation of resource management techniques in the internet of things, edge and fog computing environments," Softw., Pract. Exper., vol. 47, no. 9, pp. 1275-1296, 2017. [Online]. Available: https://doi.org/10.1002/spe.2509

[42] D. Rahbari and M. Nickray, "Scheduling of fog networks with optimized knapsack by symbiotic organisms search," in 201721 st
Conference of Open Innovations Association (FRUCT), Nov 2017, pp. 278-283.

[43] Z. Wen, R. Yang, P. Garraghan, T. Lin, J. Xu, and M. Rovatsos, "Fog orchestration for internet of things services," IEEE Internet Computing, vol. 21, no. 2, pp. 16-24, Mar 2017.

[44] O. Skarlat, M. Nardelli, S. Schulte, M. Borkowski, and P. Leitner, "Optimized iot service placement in the fog," Service Oriented Computing and Applications, vol. 11, no. 4, pp. 427-443, Dec 2017. [Online]. Available: https://doi.org/10.1007/s11761-017-0219-8

[45] H. Zhang, Y. Xiao, S. Bu, D. Niyato, F. R. Yu, and Z. Han, "Computing resource allocation in three-tier iot fog networks: A joint optimization approach combining stackelberg game and matching," IEEE Internet of Things Journal, vol. 4, no. 5, pp. 1204-1215, Oct 2017.

[46] Z. Tang, X. Zhou, F. Zhang, W. Jia, and W. Zhao, "Migration modeling and learning algorithms for containers in fog computing," IEEE Transactions on Services Computing, pp. 1-1, 2018.

[47] R. A. Thacker, K. R. Jones, C. J. Myers, and H. Zheng, "Automatic abstraction for verification of cyber-physical systems," in Proceedings of the 1st ACM/IEEE International Conference on Cyber-Physical Systems, ser. ICCPS '10. New York, NY, USA: ACM, 2010, pp. 1221. [Online]. Available: http://doi.acm.org/10.1145/1795194.1795197

[48] Z. S. Andraus and K. A. Sakallah, "Automatic abstraction and verification of verilog models," in Proceedings of the 41st Annual Design Automation Conference, ser. DAC '04. New York, NY, USA: ACM, 2004, pp. 218-223. [Online]. Available: http://doi.acm.org/10.1145/996566.996629

[49] J. Zhang, H. J. Goldsby, and B. H. Cheng, "Modular verification of dynamically adaptive systems," in Proceedings of the 8th ACM International Conference on Aspect-oriented Software Development, ser. AOSD '09. New York, NY, USA: ACM, 2009, pp. 161-172. [Online]. Available: http://doi.acm.org/10.1145/1509239.1509262

[50] S. Chaki, E. M. Clarke, A. Groce, S. Jha, and H. Veith, "Modular verification of software components in c," IEEE Transactions on Software Engineering, vol. 30, no. 6, pp. 388-402, June 2004.

[51] T. Wahl and A. Donaldson, "Replication and abstraction: Symmetry in automated formal verification,” Symmetry, vol. 2, no. 2, pp. 799-847, 2010.

[52] M. Kwiatkowska, G. Norman, and D. Parker, "Symmetry reduction for probabilistic model checking," in Computer Aided Verification, T. Ball and R. B. Jones, Eds. Berlin, Heidelberg: Springer Berlin Heidelberg, 2006, pp. 234-248.

[53] E. A. Emerson and T. Wahl, "Dynamic symmetry reduction," in Tools and Algorithms for the Construction and Analysis of Systems, N. Halbwachs and L. D. Zuck, Eds. Berlin, Heidelberg: Springer Berlin Heidelberg, 2005, pp. 382-396.

[54] L. Benalycherif and A. McIsaac, "A semantic condition for data independence and applications in hardware verification," Electronic Notes in Theoretical Computer Science, vol. 250, no. 1, pp. 39 - 54, 2009, proceedings of the Seventh International Workshop on Automated Verification of Critical Systems (AVoCS 2007). [Online]. Available: http://www.sciencedirect.com/science/article/pii/S1571066109003296

[55] L. Momtahan, "Towards a small model theorem for data independent systems in alloy," Electronic Notes in Theoretical Computer Science, vol. 128, no. 6, pp. 37 - 52, 2005, proceedings of the Fouth International Workshop on Automated Verification of Critical Systems (AVoCS 2004). [Online]. Available: http://www.sciencedirect.com/science/article/pii/S1571066105002355

[56] Chih-Chun Lee, J. R. Jiang, Chung-Yang Huang, and A. Mishchenko, "Scalable exploration of functional dependency by interpolation and incremental sat solving," in 2007 IEEE/ACM International Conference on Computer-Aided Design, Nov 2007, pp. 227-233.

[57] J.-H. R. Jiang and R. K. Brayton, "Functional dependency for verification reduction," in Computer Aided Verification, R. Alur and D. A. Peled, Eds. Berlin, Heidelberg: Springer Berlin Heidelberg, 2004, pp. 268-280.

[58] C. N. Ip and D. L. Dill, "State reduction using reversible rules," in Proceedings of the 33st Conference on Design Automation, Las Vegas, Nevada, USA, Las Vegas Convention Center, June 3-7, 1996., 1996, pp. 564-567. [Online]. Available: https://doi.org/10.1145/240518.240625

[59] C. N. Ip, "Generalized reversible rules," in Proceedings of the Second International Conference on Formal Methods in Computer-Aided Design, ser. FMCAD '98. London, UK, UK: Springer-Verlag, 1998, pp. 403-420. [Online]. Available: http://dl.acm.org/citation.cfm?id=646185.758715 
[60] S. Bensalem, M. Krichen, L. Majdoub, R. Robbana, and S. Tripakis, "A simplified approach for testing real-time systems based on action refinement," in ISoLA, ser. Revue des Nouvelles Technologies de l'Information, vol. RNTI-SM-1. Cépaduès-Éditions, 2007, pp. 191202.

[61] M. Krichen and S. Tripakis, "Conformance testing for real-time systems," Formal Methods in System Design, vol. 34, no. 3, pp. 238304, 2009.

[62] N. Bertrand, A. Stainer, T. Jéron, and M. Krichen, "A game approach to determinize timed automata," in International Conference on Foundations of Software Science and Computational Structures. Springer, Berlin, Heidelberg, 2011, pp. 245-259.

[63] N. Bertrand, T. Jéron, A. Stainer, and M. Krichen, "Off-line test selection with test purposes for non-deterministic timed automata," in International Conference on Tools and Algorithms for the Construction and Analysis of Systems. Springer, Berlin, Heidelberg, 2011, pp. 96111.

[64] M. Lahami, M. Krichen, H. Barhoumi, and M. Jmaiel, "Selective test generation approach for testing dynamic behavioral adaptations," in Testing Software and Systems - 27th IFIP WG 6.1 International Conference, ICTSS 2015, Sharjah and Dubai, United Arab Emirates, November 23-25, 2015, Proceedings, 2015, pp. 224-239. [Online]. Available: https://doi.org/10.1007/978-3-319-25945-1_14

[65] A. J. Maâlej, M. Lahami, M. Krichen, and M. Jmaïel, "Distributed and resource-aware load testing of WS-BPEL compositions," in ICEIS (2). SciTePress, 2018, pp. 29-38.

[66] M. Lahami, M. Krichen, M. Bouchakwa, and M. Jmaïel, "Using Knapsack Problem Model to Design a Resource Aware Test Architecture for Adaptable and Distributed Systems," in Proceedings of the 24th IFIP WG 6.1 International Conference Testing Software and Systems (ICTSS'12), 2012, pp. 103-118.

[67] M. Krichen and S. Tripakis, "State identification problems for timed automata," in The 17th IFIP Intl. Conf. on Testing of Communicating Systems (TestCom'05), ser. LNCS, vol. 3502. Springer, 2005.

[68] M. Krichen, State Identification. Berlin, Heidelberg: Springer Berlin Heidelberg, 2005, pp. 35-67.

[69] M. Krichen and S. Tripakis, "State identification problems for finitestate transducers," in Formal Approaches to Testing and Runtime Verification (FATES-RV'06), ser. LNCS. Springer, 2006, to appear.

[70] M. Krichen, A. J. Maâlej, and M. Lahami, "A model-based approach to combine conformance and load tests: an ehealth case study," IJCCBS, vol. 8, no. 3/4, pp. 282-310, 2018. [Online]. Available: https://doi.org/10.1504/IJCCBS.2018.096437

[71] A. J. Maâlej, M. Krichen, and M. Jmaiel, "Conformance testing of WS-BPEL compositions under various load conditions," in 36th Annual IEEE Computer Software and Applications Conference, COMPSAC 2012, Izmir, Turkey, July 16-20, 2012, 2012, p. 371. [Online]. Available: https://doi.org/10.1109/COMPSAC.2012.100

[72] A. J. Maâlej and M. Krichen, "A model based approach to combine load and functional tests for service oriented architectures." in VECoS, 2016, pp. 123-140.

[73] A. J. Malej and M. Krichen, "Study on the limitations of ws-bpel compositions under load conditions," The Computer Journal, vol. 58, no. 3, pp. 385-402, March 2015.

[74] A. J. Maâlej, M. Hamza, M. Krichen, and M. Jmaiel, "Automated significant load testing for WS-BPEL compositions," in Sixth IEEE International Conference on Software Testing, Verification and Validation, ICST 2013 Workshops Proceedings, Luxembourg, Luxembourg, March 18-22, 2013, 2013, pp. 144-153. [Online]. Available: https://doi.org/10.1109/ICSTW.2013.25

[75] A. J. Maâlej, M. Krichen, and M. Jmaiel, "Model-based conformance testing of WS-BPEL compositions," in 36th Annual IEEE Computer Software and Applications Conference Workshops, COMPSAC 2012, Izmir, Turkey, July 16-20, 2012, 2012, pp. 452-457. [Online]. Available: https://doi.org/10.1109/COMPSACW.2012.86 